\title{
On the Vocabulary of Grammar-Based Codes and the Logical Consistency of Texts
}

\author{
Łukasz Dębowski*
}

\begin{abstract}
The article presents a new interpretation for Zipf-Mandelbrot's law in natural language which rests on two areas of information theory. Firstly, we construct a new class of grammar-based codes and, secondly, we investigate properties of strongly nonergodic stationary processes. The motivation for the joint discussion is to prove a proposition with a simple informal statement: If a text of length $n$ describes $n^{\beta}$ independent facts in a repetitive way then the text contains at least $n^{\beta} / \log n$ different words, under suitable conditions on $n$. In the formal statement, two modeling postulates are adopted. Firstly, the words are understood as nonterminal symbols of the shortest grammar-based encoding of the text. Secondly, the text is assumed to be emitted by a finite-energy strongly nonergodic source whereas the facts are binary IID variables predictable in a shiftinvariant way.
\end{abstract}

Key words: excess entropy, grammar-based codes, language models, nonergodic processes, Zipf-Mandelbrot's law

${ }^{*}$ The research reported in this work was supported in part by the Australian Research Council, DP0210999, and the IST Programme of the European Community, under the PASCAL II Network of Excellence, IST-2002-506778. The material presented in Section III of this paper was published in conference communication [1].

Ł. Dębowski is with the Institute of Computer Science, Polish Academy of Sciences, ul. Ordona 21, 01-237 Warszawa, Poland (e-mail: ldebowsk@ipipan.waw.pl). 


\section{Introduction}

"If a Martian scientist sitting before his radio in Mars accidentally received from Earth the broadcast of an extensive speech [...], what criteria would he have to determine whether the reception represented the effect of animate process on Earth, or merely the latest thunderstorm on Earth? It seems that the only criteria would be the arrangement of occurrences of the elements, and the only clue to the animate origin would be this: the arrangement of the occurrences would be neither of rigidly fixed regularity such as frequently found in wave emissions of purely physical origin nor yet a completely random scattering of the same."

\section{G. K. Zipf 2, page 187]}

The aim of this paper is to present a new explanation for the distribution of words in natural language. To achieve this goal, we will consider a new class of grammar-based codes [3, 4] and we will research information-theoretic properties of strongly nonergodic stationary processes. Thus both linguists and information theorists may find this paper interesting.

The distribution of words is quite well described by the celebrated ZipfMandelbrot law [2, 5], which states that the word frequency in a text is an inverse power of the word rank. Some effort in applied probability theory has been devoted to inferring this law for various idealized settings. The most famous explanation is given by the 'monkey-typing' model. In this explanation, consecutive characters of the text are modeled as independent identically distributed (IID) variables assuming values of both letters and spaces whereas the ZipfMandelbrot law is obeyed by strings of letters delimited by spaces [5, 6]. Other published explanations involve, e.g., multiplicative processes [7] and games [8].

In this paper, we will consider the integrated version of the Zipf-Mandelbrot law, usually called Herdan's or Heaps' law in the English literature. This law says that the number of distinct words observed in a text is proportional to a power of the text length [9, 10, 11, 12]. The claim can be inferred from the Zipf-Mandelbrot law assuming certain regularity of text growth [13, 14].

The explanation of Herdan's law proposed here is based on previous partial insights [15, 16, 17, 18] and addresses two modeling challenges:

(i) Words, in the linguistic sense, can be delimited in the text even when the spaces are absent 19, 20, 21].

(ii) Texts, in the linguistic sense, refer to many facts unknown a priori to the reader but they usually do this in a consistent and repetitive way.

Our interest will be focused on proving a proposition that can be expressed in the following informal way, assuming thereafter $\beta \in(0,1)$ :

(H) If a text of length $n$ describes $n^{\beta}$ independent facts in a repetitive way then the text contains at least $n^{\beta} / \log n$ different words, under appropriate quantification over $n$.

As we will argue later in this section, some connection can also be drawn between proposition $(\mathrm{H})$ and the initial quotation from G. K. Zipf. 
So as to translate proposition $(\mathrm{H})$ into a provable statement, we will adopt a certain mathematical model of words, texts, and facts that can be motivated linguistically. The main modeling assumptions are described below. We assume that symbol $\mathbb{N}$ denotes the set of (strictly) positive integers. For a fixed countable set $\mathbb{X}$, called the alphabet, we denote the set of nonempty strings as $\mathbb{X}^{+}:=\bigcup_{n \in \mathbb{N}} \mathbb{X}^{n}$ and the set of all strings as $\mathbb{X}^{*}:=\mathbb{X}^{+} \cup\{\lambda\}$, where $\lambda$ is the empty string. The length of a string $w \in \mathbb{X}^{*}$, or $w \in \mathbb{Y}^{*}$ for any other alphabet $\mathbb{Y}$, will be written as $|w|$.

The number of words in a text: Linguists have observed that strings of characters that are repeated within the text sufficiently many times often correspond to whole words or set phrases like New York. A particularly good correspondence is obtained when word boundaries are detected using a grammarbased code that minimizes the length of a certain text encoding [19, 20, 21, 22]. For that reason, the number of words in the formalization of proposition $(\mathrm{H})$ will be modeled with the number of nonterminal symbols in such an encoding. Let us present some details of this construction.

Grammar-based codes compress strings by transforming them first into special grammars, called admissible grammars [3], and then encoding the grammars back into strings according to a fixed simple method. An admissible grammar is a context-free grammar that generates a singleton language $\{w\}$ for some string $w \in \mathbb{X}^{*}[3]$. The subset of such grammars will be denoted as $\mathcal{G}(w)$, whereas the set of admissible grammars for all strings is written as $\mathcal{G}:=\bigcup_{w \in \mathbb{X}^{*}} \mathcal{G}(w)$. If the string $w$ contains repeated substrings then some grammar in $\mathcal{G}(w)$ 'factors out' the repetitions and represents $w$ concisely.

In an admissible grammar, there is exactly one rule per nonterminal symbol and the nonterminals can be ordered so that the symbols are rewritten onto strings of strictly succeeding symbols [3, 4]. Hence, such a grammar is given by its set of production rules

$$
\mathrm{G}=\left\{\begin{array}{l}
A_{1} \rightarrow \alpha_{1}, \\
A_{2} \rightarrow \alpha_{2}, \\
\cdots, \\
A_{n} \rightarrow \alpha_{n}
\end{array}\right\}
$$

where $A_{1}$ is the start symbol, other $A_{i}$ are secondary nonterminals, and the right-hand sides of rules satisfy $\alpha_{i} \in\left(\left\{A_{i+1}, A_{i+2}, \ldots, A_{n}\right\} \cup \mathbb{X}\right)^{*}$.

A concrete example of an admissible grammar is

$$
\left\{\begin{array}{l}
A_{1} \rightarrow A_{2} A_{2} A_{4} A_{5} \text { dear_children } A_{5} A_{3} \text { all. } \\
A_{2} \rightarrow A_{3} \text { you } A_{5} \\
A_{3} \rightarrow A_{4} \text { to__ } \\
A_{4} \rightarrow \text { Good_morning } \\
A_{5} \rightarrow,_{-}
\end{array}\right\} .
$$

If we start the derivation with the symbol $A_{1}$ and follow the rewriting rules, we obtain the text of a song:

Good morning to you,

Good morning to you,

Good morning, dear children,

Good morning to all. 
In the compressions of longer texts, nonterminal symbols $A_{i}$ often correspond to words or set phrases, especially if it is also required that the nonterminals were defined as strings of only terminal symbols [21]. The latter kind of grammars will be called flat grammars.

The number of distinct nonterminal symbols in grammar (10) will be called the vocabulary size of $\mathrm{G}$ and denoted by

$$
\mathbf{V}[\mathrm{G}]:=\operatorname{card}\left\{A_{1}, A_{2}, \ldots, A_{n}\right\}=n .
$$

On the other hand, a function $\Gamma: \mathbb{X}^{+} \rightarrow \mathcal{G}$ such that $\Gamma(w) \in \mathcal{G}(w)$ for all $w \in \mathbb{X}^{+}$ is called a grammar transform [3]. In Subsection [II-C, we will construct admissibly minimal grammar transforms, which minimize the length of a specific text encoding. The vocabulary size of these transforms will be considered in the formalization of proposition $(\mathrm{H})$. The definition of admissibly minimal transforms is too technical to present right here but we may say that it resembles transforms considered by linguists [20, 21] and the transform investigated in [4], which we call Yang-Kieffer minimal. In particular, there exist admissibly minimal transforms that are flat grammar transforms.

In the second turn, we have to formulate a model of an infinitely long text that describes random facts in a repetitive way. Both the text and the set of facts repeatedly described in the text will be modeled as stochastic processes. We introduce quite a new mathematical model of human language so we devote more space for motivation.

The model of texts and facts: Let $\left(X_{i}\right)_{i \in \mathbb{Z}}$ be a stochastic process on a probability space $(\Omega, \mathfrak{J}, P)$, where variables $X_{i}: \Omega \rightarrow \mathbb{X}$ assume values from the countable alphabet $\mathbb{X}$. This process will model an infinitely long text, where $X_{i}$ are consecutive text units. We can imagine that the values of $X_{i}$ are characters if $\mathbb{X}$ is finite, or words or sentences if $\mathbb{X}$ is infinite. In most cases in this paper, we will assume that $\mathbb{X}$ is finite. On the other hand, let $Z_{k}: \Omega \rightarrow\{0,1\}, k \in \mathbb{N}$, be equidistributed IID binary variables. We will assume that the values of $Z_{k}$ are a priori unknown to the reader of the text $\left(X_{i}\right)_{i \in \mathbb{Z}}$ but can be learned from the text. Variables $Z_{k}$ will be called facts. We can imagine that the values of $Z_{k}$ are logical values $(1=$ true and $0=$ false) of certain systematically enumerated logically independent propositions.

More specifically, we will suppose that each fact $Z_{k}$ can be inferred from a half-infinite text if we start reading it from an arbitrary position. This is done to incorporate the postulate that facts are described in the text in a repetitive way. In the following, notation $X_{m: n}:=\left(X_{i}\right)_{m \leq i \leq n}$ will be used for strings of $X_{i}$, also called blocks. Blocks $X_{m: n}$ model finite texts. This definition, introduced in [17], captures what we need:

Definition 1 A stochastic process $\left(X_{i}\right)_{i \in \mathbb{Z}}$ is called strongly nonergodid 1 if there exists an IID binary process $\left(Z_{k}\right)_{k \in \mathbb{N}}$ with $P\left(Z_{k}=0\right)=P\left(Z_{k}=1\right)=\frac{1}{2}$ and functions $s_{k}: \mathbb{X}^{*} \rightarrow\{0,1\}, k \in \mathbb{N}$, such that

$$
\lim _{n \rightarrow \infty} P\left(s_{k}\left(X_{t+1: t+n}\right)=Z_{k}\right)=1, \quad \forall t \in \mathbb{Z}, \forall k \in \mathbb{N} .
$$

The motivation for functions $s_{k}$ comes from the idea that there is a fixed method of interpreting finite texts in natural language to infer facts, which is

\footnotetext{
${ }^{1} \mathrm{~A}$ not so fortunate name uncountable description process was used originally in [17].
} 
known as human language competence in linguistic jargon. Thus, any fact that is mentioned in texts in a repetitive way can be learned by text readers ultimately, regardless of their starting point. The facts that are mentioned repeatedly fall roughly into two types: (i) facts about the unchangeable objective world, which can be discovered and reported independently by successive generations of text creators, and (ii) facts about historical heritage, which undergo distributed creation, accumulation, and (partly lossy) transmission from text creators to readers.

Definition 1 is a mathematical model that ignores distinction between these two flavors of facts, except for the requirement that facts cannot change after their discovery or be forgotten after their creation. Investigating a relaxed condition is planned for a future publication. An enumeration of independent facts can also be modeled by the binary expansion of halting probability. The binary expansion of halting probability is algorithmically random and represents a large body of mathematical knowledge in its most condensed form [23, 24]. We suppose, however, that information relayed by humans in a repetitive way is mostly unrelated to this theoretical concept.

From the probabilistic point of view, a stationary process is strongly nonergodic if and only if there exists a continuous random variable $Y: \Omega \rightarrow(0,1)$ measurable with respect to the shift-invariant $\sigma$-algebra [17, Theorem 9]. Such a variable is an example of a parameter in Bayesian statistics. For instance, taking $Y=\sum_{k \in \mathbb{N}} 2^{-k} Z_{k}$ corresponds to a uniform prior on $Y$. Theorem 9 from [17] has a few consequences. Firstly, a strongly nonergodic process cannot be ergodic, or IID in particular. Secondly, it cannot be a finite-state hidden Markov process, which is a kind of processes considered in the 'monkey-typing' explanations of Zipf-Mandelbrot's law.

However, to illustrate how the concept of a strongly nonergodic process matches some preconceptions about human communication, let us consider the following example. It is simple but very different from parametric models usually considered in statistics. For a while, let the alphabet be $\mathbb{X}=\mathbb{N} \times\{0,1\}$ and let the process $\left(X_{i}\right)_{i \in \mathbb{Z}}$ have the form

$$
X_{i}:=\left(K_{i}, Z_{K_{i}}\right)
$$

where $\left(Z_{k}\right)_{k \in \mathbb{N}}$ and $\left(K_{i}\right)_{i \in \mathbb{Z}}$ are probabilistically independent whereas $\left(K_{i}\right)_{i \in \mathbb{Z}}$ is such an ergodic stationary process that $P\left(K_{i}=k\right)>0$ for every natural number $k \in \mathbb{N}$. Under such assumptions it can be demonstrated that $\left(X_{i}\right)_{i \in \mathbb{Z}}$ forms a strongly nonergodic process 2

Variables $X_{i}=\left(K_{i}, Z_{K_{i}}\right)$ can be given such a linguistic interpretation: Imagine that $\left(X_{i}\right)_{i \in \mathbb{Z}}$ is a sequence of consecutive statements extracted from an infinitely long text that describes an infinite random object $\left(Z_{k}\right)_{k \in \mathbb{N}}$ consistently. Each statement $X_{i}=(k, z)$ reveals both the address $k$ of a random bit of $\left(Z_{k}\right)_{k \in \mathbb{N}}$ and its value $Z_{k}=z$. Logical consistency of the description is reflected in this property: If two statements $X_{i}=(k, z)$ and $X_{j}=\left(k^{\prime}, z^{\prime}\right)$ describe bits of the same address $\left(k=k^{\prime}\right)$ then they always assert the same bit value $\left(z=z^{\prime}\right)$. Let us note that the pool of facts $\left(Z_{k}\right)_{k \in \mathbb{N}}$ can be viewed either as a random state of an objective world that exists prior to the text $\left(X_{i}\right)_{i \in \mathbb{Z}}$ or as historical

\footnotetext{
${ }^{2}$ In spite of a few years of acquaintance, I have not found a plausible scientific name for process (5). Probably it should be called simply the Santa Fe process because I discovered it during a visit to the Santa Fe Institute.
} 
heritage that is created on-line and memorized during generation of consecutive variables $X_{i}$. Model (5) is indifferent with respect to either interpretation.

In the formalization of proposition $(\mathrm{H})$, the number of facts described in the finite text $X_{1: n}$ will be identified with the number of $Z_{i}$ 's that may be predicted with probability at least $\delta$ given $X_{1: n}$. That is, this number will be understood as the cardinality of set

$$
U_{\delta}(n):=\left\{k \in \mathbb{N}: P\left(s_{k}\left(X_{1: n}\right)=Z_{k}\right) \geq \delta\right\},
$$

where $\delta>\frac{1}{2}$. As we will show in Subsection IV-B, the cardinality of set $U_{\delta}(n)$ is of order $n^{\beta}$ for process (5D) if variables $K_{i}$ are IID and power-law distributed,

$$
P\left(K_{i}=k\right)=k^{-1 / \beta} / \zeta\left(\beta^{-1}\right), \quad \beta \in(0,1),
$$

where $\zeta(x)=\sum_{k=1}^{\infty} k^{-x}$ is the zeta function. In contrast, it can be seen that the cardinality of $U_{\delta}(n)$ is of order $\log n$ if $\left(X_{i}\right)_{i \in \mathbb{Z}}$ is a Bernoulli process with a random parameter $Y=\sum_{k \in \mathbb{N}} 2^{-k} Z_{k}$. Note that the cardinality of $U_{\delta}(n)$ for a given process depends, to a certain extent, on the choice of functions $s_{k}$ and facts $Z_{k}$. The formalization of proposition (H) holds, however, for any choice of $s_{k}$ and $Z_{k}$ as long as (4) is satisfied.

Now we can approach the main result. Let $\mathbf{E}$ be the expectation operator and let card $A$ be the cardinality of a set $A$. We also use this concept from [25]:

Definition 2 A process $\left(X_{i}\right)_{i \in \mathbb{Z}}$ is called a finite-energy process if

$$
P\left(X_{t+|w|+1: t+|w u|}=u \mid X_{t+1: t+|w|}=w\right) \leq K c^{|u|}
$$

for all $t \in \mathbb{Z}$, all $u, w \in \mathbb{X}^{*}$, and certain constants $c<1$ and $K$, as long as $P\left(X_{t+1: t+|w|}=w\right)>0$.

It can be easily seen that stationary finite-energy processes have a positive entropy rate. Moreover, condition (8) is satisfied for processes dithered with an IID noise [25] - so it seems reasonable in modeling natural language.

Our formalization of proposition $(\mathrm{H})$ takes the following form:

Theorem 1 Let $\left(X_{i}\right)_{i \in \mathbb{Z}}$ be a stationary finite-energy strongly nonergodic process over a finite alphabet $\mathbb{X}$. Assume that inequality

$$
\liminf _{n \rightarrow \infty} \frac{\operatorname{card} U_{\delta}(n)}{n^{\beta}}>0
$$

holds for some $\beta \in(0,1), \delta \in\left(\frac{1}{2}, 1\right)$, and sets (6) where functions $s_{k}$ satisfy (4). Then

$$
\limsup _{n \rightarrow \infty} \mathbf{E}\left(\frac{\mathbf{V}\left[\Gamma\left(X_{1: n}\right)\right]}{n^{\beta}(\log n)^{-1}}\right)^{p}>0, \quad p>1,
$$

for any admissibly minimal grammar transform $\Gamma: \mathbb{X}^{+} \rightarrow \mathcal{G}$.

As we will see in Subsection IV-B, an example of a process over a finite alphabet that satisfies the premise of Theorem 1 can be constructed by stationary coding of the process (5) with $K_{i}$ satisfying (7), cf. [18]. 
Theorem 1 is closely related to two propositions pertaining to mutual information between two adjacent blocks. For a discrete stationary process $\left(X_{i}\right)_{i \in \mathbb{Z}}$, let us define the $n$-symbol entropy

$$
H(n):=H\left(X_{t+1: t+n}\right)=-\mathbf{E} \log P\left(X_{t+1: t+n}\right),
$$

where $\log$ is the natural logarithm. Denote the block mutual information as

$$
E(n):=2 H(n)-H(2 n)=I\left(X_{1: n} ; X_{n+1: 2 n}\right),
$$

called the $n$-symbol excess entropy in [26]. $E(n)$ is a convenient measure of long-range dependence in discrete-valued processes. We have:

Theorem 2 Let $\left(X_{i}\right)_{i \in \mathbb{Z}}$ be a stationary strongly nonergodic process over a finite alphabet $\mathbb{X}$. Assume that inequality (9) holds for some $\beta \in(0,1), \delta \in\left(\frac{1}{2}, 1\right)$, and sets (6) where functions $s_{k}$ satisfy (4). Then

$$
\limsup _{n \rightarrow \infty} \frac{E(n)}{n^{\beta}}>0 \text {. }
$$

Theorem 3 Let $\left(X_{i}\right)_{i \in \mathbb{Z}}$ be a stationary finite-energy process over a finite alphabet $\mathbb{X}$. Assume that inequality

$$
\liminf _{n \rightarrow \infty} \frac{E(n)}{n^{\beta}}>0
$$

holds for some $\beta \in(0,1)$. Then we have (10) for any admissibly minimal grammar transform $\Gamma: \mathbb{X}^{+} \rightarrow \mathcal{G}$.

Although Theorem 1 does not follow from Theorems 2 and 3 , we will give almost a simultaneous proof of all three propositions. A heuristic proof of Theorem 3 was outlined in [15]. This paper provides the formal proof and develops a discussion of the logically earlier Theorem 2 Because of space constraints, we do not discuss hypothetical extensions of Theorem 1 such as strong laws.

The proper discussion of the linguistic relevance of our results is also beyond the scope of this paper and will be presented in later publications. However, let us note that the conjecture $E(n) \propto n^{\beta}$ was raised for natural language by Hilberg [27]. This was his interpretation of the graph of conditional entropy in Shannon's seminal paper [28] and he supposed that $\beta \approx \frac{1}{2}$. This conjecture is little known among linguists but has evoked a discussion about 'statistical complexity' among physicists [29, 30, 31, 26]. In our opinion, Theorem 2 demonstrates that Hilberg's hypothesis can be motivated rationally, whereas Theorem 3 shows that the hypothesis implies certain empirical regularities. The initial quotation from G. K. Zipf matches these results qualitatively since processes with $E(n) \propto n^{\beta}$ differ from both regular oscillations and memoryless noise. Indeed, our preliminary experiments indicate that the vocabulary size of admissibly minimal codes is much larger for texts in natural language than for memoryless sources [16].

The further composition of this paper is as follows: Section II contains the proof of Theorems 1, 2, and 3. In Section [II] we define admissibly minimal grammar transforms and build a new class of universal grammar-based codes associated with those transforms. Section IV is a study of nonergodic stationary processes. It contains two results used to prove Theorem 2 and an exposition of a process that satisfies the assumption of Theorem 11. The article is 
supplemented with two appendices. In Appendix A, we bound the expression $2 G(n)-G(2 n)$ for a nonnegative function $G$ that has a vanishing linear rate of growth. Appendix B provides an upper bound for the expected length of a repeat in a block sampled from a finite-energy process.

\section{The proof of Theorems $1-3$}

The proof rests on several intermediate results developed later in this paper. Let $H(n)$ be the $n$-symbol entropy of the stationary process $\left(X_{i}\right)_{i \in \mathbb{Z}}$, defined in (11). The entropy rate

$$
h:=\inf _{n \in \mathbb{N}} H(n) / n=\lim _{n \rightarrow \infty} H(n) / n
$$

is another important parameter of the process [32].

Consecutively, we will use a convenient shorthand

$$
H^{U}(n):=h n+[\log 2-\eta(\delta)] \cdot \operatorname{card} U_{\delta}(n),
$$

where $\eta(p)$ denotes the entropy of distribution $(p, 1-p)$,

$$
\eta(p):=-p \log p-(1-p) \log (1-p) .
$$

By Theorem 8 from Subsection IV-A we have

$$
H(n) \geq H^{U}(n)
$$

and

$$
\lim _{n \rightarrow \infty} H(n) / n=h=\lim _{n \rightarrow \infty} H^{U}(n) / n
$$

for a strongly nonergodic process over a finite alphabet.

Consider next the $n$-symbol excess entropy $E(n)=2 H(n)-H(2 n)$. From (18) and (19) we obtain

$$
\liminf _{n \rightarrow \infty} \frac{\operatorname{card} U_{\delta}(n)}{n^{\beta}}>0 \Longrightarrow \limsup _{n \rightarrow \infty} \frac{E(n)}{n^{\beta}}>0
$$

as an instance of implication (72) from Appendix A, This proves Theorem 2 ,

Now let us proceed to prove the claims that involve grammar transforms. For an admissibly minimal grammar transform $\Gamma$, let $C=B(\Gamma(\cdot)): \mathbb{X}^{+} \rightarrow$ $\mathbb{Y}^{+}, \mathbb{Y}=\left\{0,1, \ldots, D_{Y}-1\right\}$, be the associated grammar-based code, defined in Subsection III-C (Definition 8). Denote the expected length of the code $C$ as

$$
H^{C}(n):=\mathbf{E}\left|C\left(X_{1: n}\right)\right| \log D_{Y} .
$$

This code is uniquely decodable (i.e., its extension $C^{*}:\left(u_{1}, \ldots, u_{k}\right) \mapsto$ $C\left(u_{1}\right) \ldots C\left(u_{k}\right)$ is an injection), so we have the source coding inequality

$$
H^{C}(u) \geq H(n) .
$$

Moreover, by Theorem 5 from Subsection [II-B, code $C$ is nearly universal, i.e.,

$$
\lim _{n \rightarrow \infty} H^{C}(n) / n=h=\lim _{n \rightarrow \infty} H(n) / n
$$


for any stationary finite-energy process over a finite alphabet.

Consider the expected excess length $E^{C}(n):=2 H^{C}(n)-H^{C}(2 n)$ of the code $C$. Relations (22) and (23) yield

$$
\liminf _{n \rightarrow \infty} \frac{E(n)}{n^{\beta}}>0 \Longrightarrow \limsup _{n \rightarrow \infty} \frac{E^{C}(n)}{n^{\beta}}>0
$$

as an instance of implication (71) from Appendix A,

Moreover, for a stationary finite-energy strongly nonergodic process over a finite alphabet there holds a double inequality

$$
H^{C}(u) \geq H(n) \geq H^{U}(n)
$$

and an equality of rates

$$
\lim _{n \rightarrow \infty} H^{C}(n) / n=\lim _{n \rightarrow \infty} H(n) / n=\lim _{n \rightarrow \infty} H^{U}(n) / n .
$$

Using implication (172) again, relations (25) and (26) yield respectively

$$
\liminf _{n \rightarrow \infty} \frac{\operatorname{card} U_{\delta}(n)}{n^{\beta}}>0 \Longrightarrow \limsup _{n \rightarrow \infty} \frac{E^{C}(n)}{n^{\beta}}>0 .
$$

To upper-bound the excess length of the code in terms of the vocabulary size, denote the maximal length of a (possibly overlapping) repeat in $w$ as

$$
\mathbf{L}(w):=\max \left\{|s|: w=x_{1} s y_{1}=x_{2} s y_{2} \wedge x_{1} \neq x_{2}\right\},
$$

where $s, x_{i}, y_{i} \in \mathbb{X}^{*}$. Then, by Theorem 6 (i)-(ii) from Subsection $\amalg I-C$, we have

$$
|C(u)|+|C(v)|-|C(w)| \leq W_{0} \mathbf{V}[\Gamma(w)](1+\mathbf{L}(w))
$$

for $w=u v$ and a certain constant $W_{0}$. In the following, define

$$
\begin{aligned}
& S_{n}:=\mathbf{V}\left[\Gamma\left(X_{1: 2 n}\right)\right] n^{-\beta} \log n, \\
& T_{n}:=\left(1+\mathbf{L}\left(X_{1: 2 n}\right)\right)(\log n)^{-1} .
\end{aligned}
$$

Inequality (29) and Hölder's inequality yield

$$
E^{C}(n) n^{-\beta} \leq W_{0} \mathbf{E} S_{n} T_{n} \leq W_{0}\left(\mathbf{E} S_{n}^{p}\right)^{1 / p}\left(\mathbf{E} T_{n}^{q}\right)^{1 / q}
$$

for $p, q>1$ such that $(p-1)(q-1)=1$.

Since $\mathbf{E} T_{n}^{q}$ are bounded above for a finite-energy process by Lemma 2 from Appendix B, consecutively we have

$$
\limsup _{n \rightarrow \infty} \frac{E^{C}(n)}{n^{\beta}}>0 \Longrightarrow \limsup _{n \rightarrow \infty} \mathbf{E} S_{n}^{p}>0 .
$$

Theorem 1 follows from propositions (27) and (30), whereas Theorem 3 is implied by propositions (24) and (30). 


\section{Grammar-based codes}

For the set of admissible grammars $\mathcal{G}$, a grammar-based code is a uniquely decodable code of form $C=B(\Gamma(\cdot)): \mathbb{X}^{+} \rightarrow \mathbb{Y}^{+}$, where $\Gamma: \mathbb{X}^{+} \rightarrow \mathcal{G}$ is a (string-to-)grammar transform and $B: \mathcal{G} \rightarrow \mathbb{Y}^{+}$is called a grammar(-tostring) encoder [3]. To guarantee existence of universal codes of this form, we will assume in this section that both the input and output alphabets are finite, $\mathbb{X}=\left\{0,1, \ldots, D_{X}-1\right\}$ and $\mathbb{Y}=\left\{0,1, \ldots, D_{Y}-1\right\}$ in particular.

We are interested in finding a class of nearly universal grammar-based codes for which the excess code length

$$
|C(u)|+|C(v)|-|C(u v)|
$$

can be bounded by the vocabulary size $\mathbf{V}[\Gamma(w)]$ and the maximal length (28) of a repeat in $w=u v$. Let us note that a similar bound can be obtained for the excess grammar length

$$
|\Gamma(u)|+|\Gamma(v)|-|\Gamma(u v)|
$$

of certain grammar transforms. In this expression, the Yang-Kieffer length of an admissible grammar is defined as

$$
|\mathrm{G}|:=\sum_{i}\left|\alpha_{i}\right|
$$

for grammar (11) [3]. We have:

Theorem 4 Let $\Gamma$ be a Yang-Kieffer minimal grammar transform, i.e.,

$$
|\Gamma(w)|=\min _{\mathrm{G} \in \mathcal{G}(w)}|\mathrm{G}|
$$

and $\alpha_{i} \neq \lambda$ for any secondary rule $A_{i} \rightarrow \alpha_{i}$ in $\Gamma(w)$. For any strings $u, v \in \mathbb{X}^{*}$ and $w=u v$ we have

$$
0 \leq|\Gamma(u)|+|\Gamma(v)|-|\Gamma(w)| \leq \mathbf{V}[\Gamma(w)] \mathbf{L}(w) .
$$

This result was noticed in part in [15, Theorem 3]. To motivate further constructions, let us present a proof of the right inequality.

Proof: Let the grammar $\mathrm{G}=\Gamma(w)$ for $w=u v$ be of form (11). We will split it into two grammars for $u$ and $v$, respectively

$$
\mathrm{G}_{L}=\left\{\begin{array}{l}
A_{1} \rightarrow x_{L} y_{L}, \\
A_{2} \rightarrow \alpha_{2}, \\
\ldots, \\
A_{n} \rightarrow \alpha_{n}
\end{array}\right\}, \quad \mathrm{G}_{R}=\left\{\begin{array}{l}
A_{1} \rightarrow y_{R} x_{R}, \\
A_{2} \rightarrow \alpha_{2}, \\
\ldots, \\
A_{n} \rightarrow \alpha_{n}
\end{array}\right\},
$$

where $y_{L}, y_{R} \in \mathbb{X}^{*}$ and either $\alpha_{1}=x_{L} x_{R}$ or $\alpha_{1}=x_{L} A_{i} x_{R}$ for some secondary nonterminal $A_{i}$. By minimality of $\Gamma$, each secondary nonterminal $A_{i}$ must appear at least twice on the right-hand sides of rules in $\mathrm{G}$. (Otherwise, we could find a strictly shorter grammar than $\mathrm{G}$ by deleting $A_{i}$ from the grammar.) Hence we have $\left|y_{L} y_{R}\right| \leq \mathbf{L}(w)$ and $\left|\alpha_{i}\right| \leq \mathbf{L}(w)$ for $i \geq 2$. Thus we obtain

$$
|\Gamma(u)|+|\Gamma(v)| \leq\left|\mathrm{G}_{L}\right|+\left|\mathrm{G}_{R}\right| \leq|\mathrm{G}|+n \cdot \mathbf{L}(w) .
$$

Regrouping the terms yields the right inequality in (35). 
There exists a grammar encoder $B_{\mathrm{YK}}: \mathcal{G} \rightarrow \mathbb{Y}^{+}$such that $C=B_{\mathrm{YK}}(\Gamma(\cdot))$ is a universal code for any Yang-Kieffer minimal grammar transform $\Gamma$ 3]. Unfortunately, for this encoder, it is hard to relate the excess grammar length (32) to the excess code length (31). Thus we will consider another encoder.

Subsequently, notation (11) will be reduced to

$$
\mathrm{G}=\left(\alpha_{1}, \alpha_{2}, \ldots, \alpha_{n}\right) .
$$

We will define a grammar encoder that represents grammar $\mathrm{G}$ as a string resembling list (36). This encoder yields nearly universal codes for grammar transforms that minimize the length of the code over a sufficiently large subclass of grammars (Theorem 5). Since the encoder provides nearly a homomorphism between some operations on grammars and strings, the respective codes satisfy also Theorem [6 an analogue of Theorem 4. Such codes and grammar transforms are called admissibly minimal and are defined in Subsection III-C.

\section{III-A Local encoders and minimal transforms}

The proof of Theorem 4 applies certain 'cut-and-paste' operations on grammars. For example, an operation resembling the following joining operation was used in [15] to prove the left inequality in (35):

Definition 3 For any function $f: \mathbb{U} \rightarrow \mathbb{W}^{*}$, where concatenation on domains $\mathbb{U}^{*}$ and $\mathbb{W}^{*}$ is defined, denote its extension to strings $f: \mathbb{U}^{*} \rightarrow \mathbb{W}^{*}$ as

$$
f^{*}\left(x_{1} x_{2} \ldots x_{m}\right):=f\left(x_{1}\right) f\left(x_{2}\right) \ldots f\left(x_{m}\right),
$$

where $x_{i} \in \mathbb{U}$. Next, for grammars $\mathrm{G}_{i}=\left(\alpha_{i 1}, \alpha_{i 2}, \ldots, \alpha_{i n_{i}}\right), i=1,2$, define the joining of $\mathrm{G}_{1}$ and $\mathrm{G}_{2}$ as

$$
\begin{aligned}
& \mathrm{G}_{1} \oplus \mathrm{G}_{2}:=\left(A_{2} A_{n_{1}+2}, H_{1}^{*}\left(\alpha_{11}\right), H_{1}^{*}\left(\alpha_{12}\right), \ldots, H_{1}^{*}\left(\alpha_{1 n_{1}}\right),\right. \\
& \left.H_{2}^{*}\left(\alpha_{21}\right), H_{2}^{*}\left(\alpha_{22}\right), \ldots, H_{2}^{*}\left(\alpha_{2 n_{2}}\right)\right),
\end{aligned}
$$

where $H_{1}\left(A_{j}\right)=A_{j+1}$ and $H_{2}\left(A_{j}\right)=A_{j+n_{1}+1}$ for nonterminals and $H_{1}(x)=$ $H_{2}(x)=x$ for terminals $x \in \mathbb{X}$.

We have $\mathrm{G}_{1} \oplus \mathrm{G}_{2} \in \mathcal{G}(u v)$ if $\mathrm{G}_{1} \in \mathcal{G}(u)$ and $\mathrm{G}_{2} \in \mathcal{G}(v)$.

Now we need such a grammar encoder $B: \mathcal{G} \rightarrow \mathbb{Y}^{+}$that the edit distance between $B\left(\mathrm{G}_{1} \oplus \mathrm{G}_{2}\right)$ and $B\left(\mathrm{G}_{1}\right) B\left(\mathrm{G}_{2}\right)$ is small. In the following construction, the set of positive integers $\mathbb{N}$ is treated as a generic infinite countable alphabet with concatenation $a b$, addition $a+b$, and subtraction $a-b$.

Definition 4 For the set of terminals $\mathbb{X}=\left\{0,1, \ldots, D_{X}-1\right\}, B: \mathcal{G} \rightarrow \mathbb{Y}^{+}$is called a local grammar encoder if

$$
B(\mathrm{G})=B_{S}^{*}\left(B_{N}(\mathrm{G})\right)
$$

where:

(i) the function $B_{N}: \mathcal{G} \rightarrow(\{0\} \cup \mathbb{N})^{*}$ encodes grammars as strings of integers so that the encoding of a grammar $\mathrm{G}=\left(\alpha_{1}, \alpha_{2}, \ldots, \alpha_{n}\right)$ is the string

$$
\begin{aligned}
& B_{N}(\mathrm{G}):= \\
& \quad F_{1}^{*}\left(\alpha_{1}\right) D_{X} F_{2}^{*}\left(\alpha_{2}\right) D_{X} \ldots D_{X} F_{n}^{*}\left(\alpha_{n}\right)\left(D_{X}+1\right),
\end{aligned}
$$

which employs identity transformation $F_{i}(x)=x$ for terminals $x \in \mathbb{X}$ and relative indexing $F_{i}\left(A_{j}\right)=D_{X}+1+j-i$ for nonterminals, 
(ii) the function $B_{S}:\{0\} \cup \mathbb{N} \rightarrow \mathbb{Y}^{+}$is an injection, the set $B_{S}(\{0\} \cup \mathbb{N})$ is prefix-free, and the length function $\left|B_{S}(\cdot)\right|$ is nondecreasing. - We call such $a B_{S}$ a natural number encoder.

The local encoder $B$ is uniquely decodable and the edit distance between $B\left(\mathrm{G}_{1} \oplus\right.$ $\mathrm{G}_{2}$ ) and $B\left(\mathrm{G}_{1}\right) B\left(\mathrm{G}_{2}\right)$ is small indeed. For instance, if $B\left(\mathrm{G}_{i}\right)=\gamma_{i} B_{\mathrm{S}}\left(D_{X}+1\right)$ then $B\left(\mathrm{G}_{1} \oplus \mathrm{G}_{2}\right)=\delta \gamma_{1} B_{\mathrm{S}}\left(D_{X}\right) \gamma_{2} B_{\mathrm{S}}\left(D_{X}+1\right)$ where $\delta=B_{\mathrm{S}}\left(D_{X}+2\right) B_{\mathrm{S}}\left(D_{X}+\right.$ $\left.2+\mathbf{V}\left[\mathrm{G}_{1}\right]\right) B_{\mathrm{S}}\left(D_{X}\right)$.

Subsequently, let us introduce grammar transforms that minimize the length of code $|B(\cdot)|$ over subclasses of admissible grammars. A subclass $\mathcal{J} \subset \mathcal{G}$ will be called sufficient if there exists a grammar transform $\Gamma: \mathbb{X}^{+} \rightarrow \mathcal{J}$, i.e., if $\mathcal{G}(w) \cap \mathcal{J} \neq \emptyset$ for all $w \in \mathbb{X}^{+}$.

Definition 5 For an arbitrary function $\|\cdot\|: \mathcal{G} \rightarrow\{0\} \cup \mathbb{N}$ (called later length) and a sufficient subclass $\mathcal{J}$, a grammar transform $\Gamma: \mathbb{X}^{+} \rightarrow \mathcal{J}$ will be called $a(\|\cdot\|, \mathcal{J})$-minimal grammar transform if $\|\Gamma(w)\| \leq\|\mathrm{G}\|$ for all $\mathrm{G} \in \mathcal{G}(w) \cap \mathcal{J}$ and $w \in \mathbb{X}^{+}$. If $\|\cdot\|=|B(\cdot)|$, the respective code $B(\Gamma(\cdot))$ will be called $(B, \mathcal{J})$ minimal.

The following sufficient subclasses of grammars will be discussed later.

Definition 6 We say that $\left(\alpha_{1}, \alpha_{2}, \ldots, \alpha_{n}\right)$ is a flat grammar if $\alpha_{i} \in \mathbb{X}^{+}$for $i \geq 2$. The set of flat grammars is denoted as $\mathcal{F}$. Secondly, $\mathcal{D}_{k} \subset \mathcal{F}$ denotes the class of $k$-block interleaved grammars, i.e., grammars $\left(\alpha_{1}, \alpha_{2}, \ldots, \alpha_{n}\right) \in \mathcal{F}$ where $\alpha_{i} \in \mathbb{X}^{k}$ for $i \geq 2$. Thirdly, $\mathcal{B}_{k} \subset \mathcal{D}_{k}$ stands for the set of $k$-block grammars, i.e., grammars $\left(u w, \alpha_{2}, \ldots, \alpha_{n}\right) \in \mathcal{D}_{k}$ where each $A_{2}, A_{3}, \ldots, A_{n}$ appears in the string $u \in\left(\left\{A_{2}, A_{3}, \ldots, A_{n}\right\}\right)^{*}$ whereas the string $w \in \mathbb{X}^{*}$ has length $|w|<k$, cf. [33]. Finally, we put the class of block grammars $\mathcal{B}:=\bigcup_{k>1} \mathcal{B}_{k}$ and the class of block interleaved grammars $\mathcal{D}:=\bigcup_{k \geq 1} \mathcal{D}_{k}$.

Flat grammar transforms $\Gamma: \mathbb{X}^{+} \rightarrow \mathcal{F}$ were used to detect word boundaries in the computational linguistic experiment by 21$]$.

\section{III-B Universal codes for local encoders}

Local encoders resemble the encoder considered by Neuhoff and Shields in [33], denoted here as $B_{\mathrm{NS}}$. The authors have established that any $\left(B_{\mathrm{NS}}, \mathcal{B}\right)$-minimal code is universal for the class of block grammars $\mathcal{B}$, and we will use this fact to prove that certain codes employing local encoders are nearly universal. The main difference between the encoder $B_{\mathrm{NS}}$ and a local encoder is that $B_{\mathrm{NS}}$ encodes a nonterminal $A_{i}$ as a string of length $\log _{D_{Y}} \mathbf{V}[\mathrm{G}]$ whereas the local encoder uses a string of length $\left|B_{\mathrm{S}}\left(D_{X}+i\right)\right|$. This is not a big difference so we can prove the following proposition using some results of [33].

Theorem 5 For the set of terminals $\mathbb{X}=\left\{0,1, \ldots, D_{X}-1\right\}$ and the output alphabet $\mathbb{Y}=\left\{0,1, \ldots, D_{Y}-1\right\}$, let $B: \mathcal{G} \rightarrow \mathbb{Y}^{+}$be a local grammar encoder (38) that satisfies

$$
\limsup _{n \rightarrow \infty}\left|B_{S}(n)\right| / \log _{D_{Y}} n=1 .
$$

Then for any sufficient subclass of grammars $\mathcal{J} \supset \mathcal{B}$, every $(B, \mathcal{J})$-minimal code $C$ is nearly strongly universal, i.e.,

$$
\limsup _{n \rightarrow \infty} \frac{\left|C\left(X_{1: n}\right)\right| \log D_{Y}}{n} \leq h \quad \text { a.s. }
$$


for any stationary ergodic process $\left(X_{k}\right)_{k \in \mathbb{Z}}$ over the alphabet $\mathbb{X}$ with an entropy rate $h>03$

Remark 1: A natural number encoder $B_{\mathrm{S}}$ such that (39) holds can be chosen,

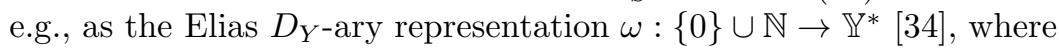

$$
|\omega(n)|= \begin{cases}1 & \text { if } n<D_{Y}, \\ \left|\omega\left(\left\lfloor\log _{D_{Y}} n\right\rfloor\right)\right|+\left\lfloor\log _{D_{Y}} n\right\rfloor+1 & \text { if } n \geq D_{Y} .\end{cases}
$$

Remark 2: Claim (40) may be generalized to finite-energy processes as follows, cf. [35]. Let $\left(X_{k}\right)_{k \in \mathbb{Z}}$ be a stationary finite-energy process over the alphabet $\mathbb{X}$ and let $h_{F}$ be the entropy rate of the process's random ergodic measure, viz. (55) and (57). Firstly, from the finite-energy property and the ShannonMcMillan-Breiman theorem it follows that $h_{F}>0$. Hence, the strong ergodic decomposition theorem [36, a statement in the proof of Theorem 9.12] and the claim of Theorem [5imply

$$
\limsup _{n \rightarrow \infty} \frac{\left|C\left(X_{1: n}\right)\right| \log D_{Y}}{n} \leq h_{F} \quad \text { a.s. }
$$

Secondly, since $0 \leq\left|C\left(X_{1: n}\right)\right| \leq K n$ for a $K>0$, inequality (41) implies

$$
\lim _{n \rightarrow \infty} \mathbf{E}\left(\frac{\left|C\left(X_{1: n}\right)\right| \log D_{Y}}{n}\right)=h
$$

by equality $h=\mathbf{E} h_{F}$ [37] and the inverse Fatou lemma.

Proof: Consider a sequence of $k$-block grammar transforms $\Gamma_{k}: \mathbb{X}^{+} \rightarrow \mathcal{B}_{k}$, $k \geq 1$. Because $\left|B_{\mathrm{S}}(\cdot)\right|$ is nondecreasing, we have

$$
\left|B\left(\Gamma_{k}(w)\right)\right| \leq \alpha k \mathbf{V}\left[\Gamma_{k}(w)\right]+\frac{n}{k}\left|B_{\mathrm{S}}\left(D_{X}+\mathbf{V}\left[\Gamma_{k}(w)\right]\right)\right|
$$

for an $\alpha>0$. On the other hand, for an $\epsilon>0$ and a stationary ergodic process $\left(X_{k}\right)_{k \in \mathbb{Z}}$ with an entropy rate $h$, let $k(n)$ be the largest integer $k$ satisfying $k e^{k(h+\epsilon)} \leq n$. Neuhoff and Shields showed in [33] that, for $h \geq 0$,

$$
\begin{aligned}
& \limsup _{n \rightarrow \infty} \max _{w \in \mathbb{X}^{n}} \frac{\log \mathbf{V}\left[\Gamma_{k(n)}(w)\right]}{k(n)} \leq h+2 \epsilon, \\
& \lim _{n \rightarrow \infty} \mathbf{V}\left[\Gamma_{k(n)}\left(X_{1: n}\right)\right] \cdot k(n) / n=0 \quad \text { a.s. }
\end{aligned}
$$

Notice that $\lim _{n} k(n)=\infty$. Hence $\lim _{n} \mathbf{V}\left[\Gamma_{k(n)}\left(X_{1: n}\right)\right]=\infty$ a.s. for $h>0$ by [38, Theorem 2 on page 912]. Moreover, (39) holds, and thus we obtain

$$
\limsup _{n \rightarrow \infty} \frac{\mid B\left(\Gamma_{k(n)}\left(X_{1: n}\right) \mid \log D_{Y}\right.}{n} \leq h+2 \epsilon \quad \text { a.s. }
$$

for $h>0$. Hence any $(B, \mathcal{J})$-minimal code is nearly strongly universal.

\footnotetext{
${ }^{3}$ This theorem was mistakenly stated in [1] without the assumption $h>0$.
} 


\section{III-C Bounds for the vocabulary size}

Now we will derive the analogue of Theorem 4 for some minimal grammarbased codes that use local grammar encoders. Firstly, the code lengths are almost subadditive. Secondly, the excess code lengths are dominated by the vocabulary size multiplied by the length of the longest repeat. To show this, we will introduce a few other operations on grammars.

Definition 7 Consider a grammar

$$
\mathrm{G}=\left(\alpha_{1}, \alpha_{2}, \ldots, \alpha_{n}\right) \in \mathcal{G}(w) .
$$

For $0 \leq p, q \leq|w|$ and $p+q=|w|$, let the strings $u, v \in \mathbb{X}^{*}$ satisfy $p=|u|$, $q=|v|$ and $u v=w$. Define then the left and right croppings of $\mathrm{G}$ as

$$
\begin{aligned}
& \mathbb{L}_{p} G:=\left(x_{L} y_{L}, \alpha_{2}, \ldots, \alpha_{n}\right) \in \mathcal{G}(u), \\
& \mathbb{R}_{q} G:=\left(y_{R} x_{R}, \alpha_{2}, \ldots, \alpha_{n}\right) \in \mathcal{G}(v),
\end{aligned}
$$

where $y_{L}, y_{R} \in \mathbb{X}^{*}$ and either $\alpha_{1}=x_{L} x_{R}$ or $\alpha_{1}=x_{L} A_{i} x_{R}$ for some secondary nonterminal $A_{i}$.

The expansion $\langle\alpha\rangle_{\mathrm{F}}$ of a string $\alpha \in\left(\left\{A_{1}, A_{2}, A_{3}, \ldots, A_{n}\right\} \cup \mathbb{X}\right)^{*}$ with respect to a subset of rules $\mathrm{F} \subset \mathrm{G}$ of the grammar (43) is the unique element of the language generated by grammar $\left\{A_{0} \rightarrow \alpha\right\} \cup \mathrm{F}$ with the start symbol $A_{0}$, cf. [4]. Define then

(i) the nonterminal deleting

$$
\begin{aligned}
\mathbb{U}_{i} \mathrm{G}:= & \left(\phi_{i}\left(\alpha_{1}\right), \phi_{i}\left(\alpha_{2}\right), \ldots \phi_{i}\left(\alpha_{i-1}\right),\right. \\
& \left.\phi_{i}\left(\alpha_{i+1}\right), \phi_{i}\left(\alpha_{i+2}\right), \ldots \phi_{i}\left(\alpha_{n}\right)\right),
\end{aligned}
$$

where $\phi_{i}(\alpha)=H_{i}^{*}\left(\langle\alpha\rangle_{\left\{A_{i} \rightarrow \alpha_{i}\right\}}\right), H_{i}\left(A_{j}\right)=A_{j-1}$ for $j>i, H_{i}\left(A_{j}\right)=A_{j}$ for $j<i, H_{i}(x)=x$ for $x \in \mathbb{X}$, and $2 \leq i \leq n$,

(ii) the flattening

$$
\mathbb{F G}:=\left(\alpha_{1},\left\langle\alpha_{2}\right\rangle_{\mathrm{G}},\left\langle\alpha_{3}\right\rangle_{\mathrm{G}}, \ldots,\left\langle\alpha_{n}\right\rangle_{\mathrm{G}}\right)
$$

(iii) and the secondary part

$$
\mathbb{S G}:=\left(\lambda, \alpha_{2}, \alpha_{3}, \ldots, \alpha_{n}\right) .
$$

Theorem 6 For the set of terminals $\mathbb{X}=\left\{0,1, \ldots, D_{X}-1\right\}$, let $B: \mathcal{G} \rightarrow \mathbb{Y}^{+}$be a local grammar encoder (38). Introduce constants

$$
W_{n}:=\left|B_{S}\left(D_{X}+1+n\right)\right| .
$$

Let $\Gamma$ be a $(\|\cdot\|, \mathcal{J})$-minimal grammar transform for the length $\|\cdot\|=|B(\cdot)|$. Consider the code $C=B(\Gamma(\cdot))$ and strings $u, v, w \in \mathbb{X}^{+}$.

(i) If $\mathrm{G} \in \mathcal{J} \Longrightarrow \mathbb{L}_{p} \mathrm{G}, \mathbb{R}_{q} \mathrm{G}, \mathbb{U}_{i} \mathrm{G} \in \mathcal{J}$ for all valid $p, q$, $i$ then

$$
\begin{aligned}
|C(u)|,|C(v)| & \leq|C(u v)|+W_{0} \mathbf{L}(u v), \\
|C(u)|+|C(v)|-|C(u v)| & \leq\|\mathbb{S} \Gamma(u v)\|+W_{0} \mathbf{L}(u v) .
\end{aligned}
$$


(ii) If $\mathrm{G} \in \mathcal{J} \Longrightarrow \mathbb{F G}, \mathbb{U}_{i} \mathrm{G} \in \mathcal{J}$ for all valid $i$ then

$$
\|\mathbb{S} \Gamma(w)\|+W_{0} \mathbf{L}(w) \leq W_{0} \mathbf{V}[\Gamma(w)](1+\mathbf{L}(w)) .
$$

(iii) If $\mathrm{G}_{1}, \mathrm{G}_{2} \in \mathcal{J} \Longrightarrow \mathrm{G}_{1} \oplus \mathrm{G}_{2} \in \mathcal{J}$ then

$$
\begin{aligned}
|C(u)|+|C(v)|-|C(u v)| & \\
& \geq-W_{0}-W_{1}-W_{\mathbf{V}[\Gamma(u)]+1} .
\end{aligned}
$$

Remark: In particular, the premises of proposition (i)-(ii) are satisfied for $\mathcal{J}=\mathcal{G}, \mathcal{F}, \mathcal{D}, \mathcal{D}_{k}$ whereas the premise of proposition (iii) is satisfied for $\mathcal{J}=\mathcal{G}$ Moreover, inequalities (46) and (47) imply together inequality (29), which is an analogue of (35) 4

Proof: If $\mathrm{G} \in \mathcal{J} \Longrightarrow \mathbb{U}_{i} \mathrm{G} \in \mathcal{J}$ for all valid $i$ then each secondary nonterminal $A_{i}$ must appear at least twice on the right-hand sides of rules in $\Gamma(w)$ whereas the right-hand side of rule $\left(A_{i} \rightarrow \alpha_{i}\right) \in \Gamma(w)$ may not be empty. (Otherwise, we would obtain $\|\Gamma(w)\|>\left\|\mathbb{U}_{i} \Gamma(w)\right\| \in \mathcal{J}$ because $\left|B_{\mathrm{S}}(n)\right|>0$ and $\left|B_{\mathrm{S}}(\cdot)\right|$ is nondecreasing.) Hence we have $\left|\left\langle\alpha_{i}\right\rangle_{\mathrm{G}}\right| \leq \mathbf{L}(w)$ for $i \geq 2$. This result is used to prove propositions (i) and (ii) below.

(i) Set $p=|u|, q=|v|$, and $w=u v$. The claimed inequalities follow from

$$
\begin{aligned}
& \|\Gamma(w)\|+W_{0} \mathbf{L}(w) \geq\left\|\mathbb{L}_{p} \Gamma(w)\right\| \geq\|\Gamma(u)\|, \\
& \|\Gamma(w)\|+W_{0} \mathbf{L}(w) \geq\left\|\mathbb{R}_{q} \Gamma(w)\right\| \geq\|\Gamma(v)\|,
\end{aligned}
$$

and

$$
\begin{aligned}
\left\|\mathbb{L}_{p} \Gamma(w)\right\|+ & \left\|\mathbb{R}_{q} \Gamma(w)\right\| \\
& \leq\|\Gamma(w)\|+\|\mathbb{S} \Gamma(w)\|+W_{0} \mathbf{L}(w) .
\end{aligned}
$$

(ii) The claim is entailed by $\|\mathbb{S} \Gamma(w)\| \leq\|\operatorname{SF} \Gamma(w)\|$ and

$$
\|\operatorname{SF} \Gamma(w)\| \leq W_{0}(\mathbf{V}[\Gamma(w)]-1)(1+\mathbf{L}(w))+W_{0} .
$$

(iii) The result is implied by $\|\Gamma(u v)\| \leq\|\Gamma(u) \oplus \Gamma(v)\|$ and

$$
\left\|\mathrm{G}_{1} \oplus \mathrm{G}_{2}\right\| \leq W_{1}+W_{\mathbf{V}\left[\mathrm{G}_{1}\right]+1}+W_{0}+\left\|\mathrm{G}_{1}\right\|+\left\|\mathrm{G}_{2}\right\|,
$$

where $\mathrm{G}_{1}=\Gamma(u)$ and $\mathrm{G}_{2}=\Gamma(v)$.

The strengths of Theorems [5] and 6 (i)-(ii) can be combined for the following class of codes and grammar transforms:

Definition 8 A grammar transform $\Gamma: \mathbb{X}^{+} \rightarrow \mathcal{G}$, where $\mathbb{X}=\left\{0,1, \ldots, D_{X}-1\right\}$, and the associated code $C=B(\Gamma(\cdot)): \mathbb{X}^{+} \rightarrow \mathbb{Y}^{+}$, where $\mathbb{Y}=\left\{0,1, \ldots, D_{Y}-1\right\}$, are called admissibly minimal if

(i) $\Gamma$ is a $(|B(\cdot)|, \mathcal{J})$-minimal grammar transform, where

(ii) $B: \mathcal{G} \rightarrow \mathbb{Y}^{+}$is a local grammar encoder (38) that satisfies (39),

(iii) $\mathcal{J} \supset \mathcal{B}$ for the subclass of block grammars $\mathcal{B}$,

(iv) $\mathrm{G} \in \mathcal{J} \Longrightarrow \mathbb{F G}, \mathbb{L}_{p} \mathrm{G}, \mathbb{R}_{q} \mathrm{G}, \mathbb{U}_{i} \mathrm{G} \in \mathcal{J}$ for all valid $p, q, i$.

Remark: In particular, we may take $\mathcal{J}=\mathcal{G}, \mathcal{F}, \mathcal{D}$.

\footnotetext{
${ }^{4}$ Propositions (i) and (ii) were mistakenly stated in [1] without the condition $\mathbb{U}_{i} G \in \mathcal{J}$.
} 


\section{Strongly nonergodic processes}

In this section we explore stationary processes rather than codes. The main goal is to demonstrate equality (19) and inequality (18) for strongly nonergodic processes over a finite alphabet. The proofs are given in Subsection IV-A. This is followed by a construction of a process that satisfies the assumption of Theorem 1 given in Subsection IV-B.

\section{IV-A A bound for the number of facts}

For this subsection we need a measure-theoretic generalization of mutual information, cf. 39]. For a probability space $(\Omega, \mathfrak{J}, P)$, a partition of the $\sigma$-algebra $\mathfrak{J} \subset 2^{\Omega}$ is a finite set of events $\left\{B_{j}\right\}_{j=1}^{J}$ such that $B_{j} \in \mathfrak{J}, B_{i} \cap B_{j}=\emptyset$, and $\bigcup_{j=1}^{J} B_{j}=\Omega$. We define mutual information between partitions $\alpha=\left\{A_{i}\right\}_{i=1}^{I}$ and $\beta=\left\{B_{j}\right\}_{j=1}^{J}$ with respect to probability measure $P$ as

$$
I_{P}(\alpha ; \beta):=\sum_{i=1}^{I} \sum_{j=1}^{J} P\left(A_{i} \cap B_{j}\right) \log \frac{P\left(A_{i} \cap B_{j}\right)}{P\left(A_{i}\right) P\left(B_{j}\right)},
$$

where $0 \log 0 / x:=0$.

Now, let $\mathfrak{A}, \mathfrak{B}$, and $\mathfrak{C}$ be subalgebras of $\sigma$-algebra $\mathfrak{J}$. That is, $\{\emptyset, \Omega\} \subset$ $\mathfrak{A}, \mathfrak{B}, \mathfrak{C} \subset \mathfrak{J}$ as well as $\mathfrak{A}, \mathfrak{B}$, and $\mathfrak{C}$ are closed w.r.t. operations $\cap, \cup$, and $\backslash$. Moreover let the random variable $P(A \| \mathfrak{C})$ be the conditional probability of event $A \in \mathfrak{J}$ w.r.t. the smallest $\sigma$-algebra containing $\mathfrak{C}$ [40, Section 33]. We may extend the concepts of conditional mutual information, mutual information, conditional entropy, and entropy respectively as

$$
\begin{aligned}
I(\mathfrak{A} ; \mathfrak{B} \mid \mathfrak{C}) & :=\sup _{\alpha \subset \mathfrak{A}, \beta \subset \mathfrak{B}} \mathbf{E} I_{P(\cdot \| \mathfrak{C})}(\alpha ; \beta), \\
I(\mathfrak{A} ; \mathfrak{B}) & :=I(\mathfrak{A} ; \mathfrak{B} \mid\{\emptyset, \Omega\}) \\
H(\mathfrak{A} \mid \mathfrak{C}) & :=I(\mathfrak{A} ; \mathfrak{A} \mid \mathfrak{C}) \\
H(\mathfrak{A}) & :=I(\mathfrak{A} ; \mathfrak{A} \mid\{\emptyset, \Omega\})
\end{aligned}
$$

where we write $\beta=\left\{B_{j}\right\}_{j=1}^{J} \subset \mathfrak{B}$ if and only if all $B_{j} \in \mathfrak{B}$, cf. [17, 41, 42]. These concepts generalize the corresponding definitions for random variables. If we consider discrete random variables $Y_{i}$ and the smallest subalgebras $\mathfrak{A}_{i} \subset \mathfrak{J}$ such that all events of form $\left(Y_{i}=y_{i}\right)$ belong to $\mathfrak{A}_{i}$, then $I\left(Y_{1} ; Y_{2} \mid Y_{3}\right)=I\left(\mathfrak{A}_{1} ; \mathfrak{A}_{2} \mid \mathfrak{A}_{3}\right)$, $I\left(Y_{1} ; Y_{2}\right)=I\left(\mathfrak{A}_{1} ; \mathfrak{A}_{2}\right), H\left(Y_{1} \mid Y_{3}\right)=H\left(\mathfrak{A}_{1} \mid \mathfrak{A}_{3}\right)$, and $H\left(Y_{1}\right)=I\left(\mathfrak{A}_{1}\right)$. Moreover, quantities (50)-(53) satisfy familiar chain rules and enjoy certain continuity [41], [42], [17, Theorems 1 and 2].

Consider a stationary process $\left(X_{i}\right)_{i \in \mathbb{Z}}$, where $X_{i}:(\Omega, \mathfrak{J}) \rightarrow(\mathbb{X}, \mathfrak{X})$. Let $\left(\mathbb{X}^{\mathbb{Z}}, \mathfrak{X}^{\mathbb{Z}}\right)$ be the measurable space of double infinite sequences. For the shift transformation $T: \mathbb{X}^{\mathbb{Z}} \ni\left(x_{k}\right)_{k \in \mathbb{Z}} \mapsto\left(x_{k+1}\right)_{k \in \mathbb{Z}} \in \mathbb{X}^{\mathbb{Z}}$, where $x_{k} \in \mathbb{X}$, define the shift-invariant algebra $\mathfrak{I}_{\mathbb{X}}:=\left\{A \in \mathfrak{X}^{\mathbb{Z}}: T A=A\right\}$. Let $(\mathbb{S}, \mathfrak{S})$ be the measurable space of stationary probability measures on $\left(\mathbb{X}^{\mathbb{Z}}, \mathfrak{X}^{\mathbb{Z}}\right)$ (i.e., $\mu \circ T=\mu$ for $\mu \in \mathbb{S}$ ) and let $(\mathbb{E}, \mathfrak{E}) \subset(\mathbb{S}, \mathfrak{S})$ be the subspace of ergodic measures (i.e., $\mu(A) \in\{0,1\}$ for $\mu \in \mathbb{E}$ and $\left.A \in \mathfrak{I}_{\mathbb{X}}\right)$. Precisely, $\mathfrak{S}$ and $\mathfrak{E}$ are defined as the smallest $\sigma$-algebras containing all cylinder sets $\{\mu \in \mathbb{S}: \mu(A) \leq r\}$ and $\{\mu \in \mathbb{E}: \mu(A) \leq r\}, A \in \mathfrak{X}^{\mathbb{Z}}$, $r \in \mathbb{R}$, respectively. 
For an arbitrary measure $\mu \in \mathbb{S}$, let us denote its $n$-symbol entropy and entropy rate

$$
\begin{aligned}
H_{\mu}(n) & :=-\mathbf{E} \log \mu\left(\xi_{t+1: t+n}\right), \\
h_{\mu} & :=\lim _{n \rightarrow \infty} H_{\mu}(n) / n,
\end{aligned}
$$

where $\xi_{i}: \mathbb{X}^{\mathbb{Z}} \ni\left(x_{k}\right)_{k \in \mathbb{Z}} \mapsto x_{i} \in \mathbb{X}$ are random variables on the space of $\left(\mathbb{X}^{\mathbb{Z}}, \mathfrak{X}^{\mathbb{Z}}\right)$. In particular, $H_{\mu}(n)=H(n)$ and $h_{\mu}=h$ when $\mu=P\left(\left(X_{k}\right)_{k \in \mathbb{Z}} \in \cdot\right)$.

Now we will consider $n$-symbol entropy and entropy rate of another measure associated with the stationary process. Let us put

$$
\mathfrak{F}:=\left(X_{i}\right)_{i \in \mathbb{Z}}^{-1}\left(\mathfrak{I}_{\mathbb{X}}\right) \subset \mathfrak{J} .
$$

According to the ergodic decomposition theorem [36, Theorems 9.10-12], if $\mathbb{X}$ is countable, then there exists a random ergodic measure $F:(\Omega, \mathfrak{F}) \rightarrow(\mathbb{E}, \mathfrak{E})$ such that

$$
F(A)=P\left(\left(X_{i}\right)_{i \in \mathbb{Z}} \in A \| \mathfrak{F}\right)
$$

for all $A \in \mathfrak{X}^{\mathbb{Z}}$. Having introduced this measure, we obtain:

Theorem 7 For a stationary process $\left(X_{i}\right)_{i \in \mathbb{Z}}$ over a finite alphabet $\mathbb{X}$,

$$
H(n)=I\left(X_{1: n} ; \mathfrak{F}\right)+\mathbf{E} H_{F}(n),
$$

where $\mathbf{E} H_{F}(n) \geq h n$.

Proof: We have $H(n)=H\left(X_{1: n}\right)$ and $\mathbf{E} H_{F}(n)=H\left(X_{1: n} \mid \mathfrak{F}\right)$ by (57). Hence (58) follows by the chain rule $H(\mathfrak{A})=I(\mathfrak{A} ; \mathfrak{B})+H(\mathfrak{A} \mid \mathfrak{B})$ [42, Section 3.6], [17, Theorem 2(ii)]. On the other hand, inequality $\mathbf{E} H_{F}(n) \geq h n$ follows from $H_{\mu}(n) \geq h_{\mu} n$ and equality $h=\mathbf{E} h_{F}$ derived by [37] for stationary processes over a finite alphabet.

This yields the needed results:

Theorem 8 For a stationary strongly nonergodic process $\left(X_{i}\right)_{i \in \mathbb{Z}}$ over a finite alphabet $\mathbb{X}$, inequality (18) and equality (19) are satisfied for $\delta \in\left(\frac{1}{2}, 1\right)$.

Proof: By continuity of mutual information [42, Section 2.2], [17, Theorems $1(\mathrm{v})$ and $2(\mathrm{i})]$,

$$
\begin{aligned}
I\left(X_{1: n} ;\left(Z_{k}\right)_{k \in \mathbb{N}}\right) & =\lim _{k \rightarrow \infty} I\left(X_{1: n} ; Z_{1: k}\right) \\
& =\sum_{k=1}^{\infty} I\left(X_{1: n} ; Z_{k} \mid Z_{1: k-1}\right) .
\end{aligned}
$$

On the other hand,

$$
\begin{aligned}
I\left(X_{1: n} ; Z_{k} \mid Z_{1: k-1}\right) & =H\left(Z_{k} \mid Z_{1: k-1}\right)-H\left(Z_{k} \mid X_{1: n}, Z_{1: k-1}\right) \\
& \geq \log 2-H\left(Z_{k} \mid s_{k}\left(X_{1: n}\right)\right) \\
& \geq \log 2-\eta\left(P\left(s_{k}\left(X_{1: n}\right)=Z_{k}\right)\right)
\end{aligned}
$$


by the Fano inequality $H\left(Y_{1} \mid Y_{2}\right) \leq \eta\left(P\left(Y_{1}=Y_{2}\right)\right)$ for a binary variable $Y_{2}$ 32, Theorem 2.11.1]. Restricting the summation in (59) to $k \in U_{\delta}(n)$ yields

$$
I\left(X_{1: n} ;\left(Z_{k}\right)_{k \in \mathbb{N}}\right) \geq[\log 2-\eta(\delta)] \cdot \operatorname{card} U_{\delta}(n)
$$

because $\eta(\delta) \geq \eta\left(P\left(s_{k}\left(X_{1: n}\right)=Z_{k}\right)\right)$ for $\delta \geq \frac{1}{2}$ and $k \in U_{\delta}(n)$. Since all events of form $\left(Z_{k}=0\right)$ and $\left(Z_{k}=1\right)$ belong to the completion of algebra $\mathfrak{F}$ by [17, Theorem 9], we have $I\left(X_{1: n} ; \mathfrak{F}\right) \geq I\left(X_{1: n} ;\left(Z_{k}\right)_{k \in \mathbb{N}}\right)$ by the data processing inequality [17, Theorem 1(iv)]. In the following, by Theorem 7 we obtain

$$
\begin{aligned}
H(n) & \geq h n+I\left(X_{1: n} ; \mathfrak{F}\right) \\
& \geq h n+I\left(X_{1: n} ;\left(Z_{k}\right)_{k \in \mathbb{N}}\right) \geq H^{U}(n) .
\end{aligned}
$$

By $H(n) \geq H^{U}(n) \geq h n$, (19) holds as well.

\section{IV-B An example of a process}

In this subsection we will present a process that satisfies the assumptions of Theorem 1. The process will be denoted as $\left(\bar{Y}_{i}\right)_{i \in \mathbb{Z}}$ and will be given by stationary coding of the process (5). The requirements for the process $\left(\bar{Y}_{i}\right)_{i \in \mathbb{Z}}$ are as follows:

(a) $\left(\bar{Y}_{i}\right)_{i \in \mathbb{Z}}$ is a process over a finite alphabet $\mathbb{Y}$,

(b) $\left(\bar{Y}_{i}\right)_{i \in \mathbb{Z}}$ is stationary,

(c) $\left(\bar{Y}_{i}\right)_{i \in \mathbb{Z}}$ has finite energy, and

(d) there exists an IID binary process $\left(\bar{Z}_{k}\right)_{k \in \mathbb{N}}$ with $\bar{P}\left(\bar{Z}_{k}=0\right)=\bar{P}\left(\bar{Z}_{k}=\right.$ 1) $=\frac{1}{2}$, and functions $\bar{s}_{k}: \mathbb{Y}^{*} \rightarrow\{0,1\}, k \in \mathbb{N}$, such that

$$
\lim _{n \rightarrow \infty} \bar{P}\left(\bar{s}_{k}\left(\bar{Y}_{t+1: t+n}\right)=\bar{Z}_{k}\right)=1, \quad \forall t \in \mathbb{Z}, \forall k \in \mathbb{N},
$$

and

$$
\liminf _{n \rightarrow \infty} \frac{\operatorname{card} \bar{U}_{\bar{\delta}}(n)}{n^{\beta}}>0
$$

for a certain $\beta \in(0,1)$, all $\bar{\delta} \in\left(\frac{1}{2}, 1\right)$, and sets

$$
\bar{U}_{\bar{\delta}}(n):=\left\{k \in \mathbb{N}: \bar{P}\left(\bar{s}_{k}\left(\bar{Y}_{1: n}\right)=\bar{Z}_{k}\right) \geq \bar{\delta}\right\} .
$$

The analogues of properties (b)-(d), but not (a), are satisfied by the process (5) with $K_{i}$ being IID and satisfying (7). For example, let us derive (9). We will write $u \sqsubseteq v$ when an infinite sequence or a string $v$ contains a string $u$ as a substring. For $\mathbb{X}=\mathbb{N} \times\{0,1\}$ and $v \in \mathbb{X}^{\mathbb{Z}} \cup \mathbb{X}^{*}$, define the predictors $s_{k}$ as

$$
s_{k}(v):= \begin{cases}0 & \text { if }(k, 0) \sqsubseteq v \text { and }(k, 1) \nsubseteq v, \\ 1 & \text { if }(k, 1) \sqsubseteq v \text { and }(k, 0) \nsubseteq v, \\ 2 & \text { else. }\end{cases}
$$


We have $Z_{k}=s_{k}\left(\left(X_{i}\right)_{i \in \mathbb{Z}}\right)$ almost surely. Then let $U_{\delta}(n)$ be the set of well predictable facts, defined in (6). In view of equality

$$
\begin{aligned}
P\left(s_{k}\left(X_{1: n}\right)=Z_{k}\right) & =P\left(K_{i}=k \text { for some } i \in\{1, \ldots, n\}\right) \\
& =1-\left[1-P\left(K_{i}=k\right)\right]^{n},
\end{aligned}
$$

we have $k \in U_{\delta}(n)$ if and only if $P\left(K_{i}=k\right) \geq 1-(1-\delta)^{1 / n}$. This yields

$$
U_{\delta}(n) \supset\left\{k \in \mathbb{N}: P\left(K_{i}=k\right) \geq-n^{-1} \log (1-\delta)\right\}
$$

by inequality $1-x^{1 / n} \leq-n^{-1} \log x$ for $x>0$. Hence

$$
\operatorname{card} U_{\delta}(n) \geq\left[\frac{n}{-\zeta\left(\beta^{-1}\right) \log (1-\delta)}\right]^{\beta} .
$$

In particular, (9) is satisfied.

Now we have to explain what stationary coding is. Firstly, consider a function $f: \mathbb{X} \rightarrow \mathbb{Y}^{*}$ that maps single symbols into strings. We define its extension to double infinite sequences $f^{\mathbb{Z}}: \mathbb{X}^{\mathbb{Z}} \rightarrow \mathbb{Y}^{\mathbb{Z}} \cup\left(\mathbb{Y}^{*} \times \mathbb{Y}^{*}\right)$ as

$$
f^{\mathbb{Z}}\left(\left(x_{i}\right)_{i \in \mathbb{Z}}\right):=\ldots f\left(x_{-1}\right) f\left(x_{0}\right) \cdot f\left(x_{1}\right) f\left(x_{2}\right) \ldots
$$

where $x_{i} \in \mathbb{X}$. (The bold-face dot separates the 0 -th and the first symbol.) Secondly, for the shift operation $T\left(\left(x_{i}\right)_{i \in \mathbb{Z}}\right):=\left(x_{i+1}\right)_{i \in \mathbb{Z}}=\ldots x_{0} x_{1} \cdot x_{2} x_{3} \ldots$, a measure $\mu$ on $\left(\mathbb{X}^{\mathbb{Z}}, \mathfrak{X}^{\mathbb{Z}}\right)$ is called asymptotically mean stationary (AMS) if limits

$$
\bar{\mu}(A)=\lim _{n \rightarrow \infty} \frac{1}{n} \sum_{i=0}^{n-1} \mu \circ T^{-i}(A)
$$

exist for all $A \in \mathfrak{X}^{\mathbb{Z}}$, cf. [43]. The limit $\bar{\mu}$, if it exists as a total function $\mathfrak{X}^{\mathbb{Z}} \rightarrow \mathbb{R}$, forms a stationary measure on $\left(\mathbb{X}^{\mathbb{Z}}, \mathfrak{X}^{\mathbb{Z}}\right)$, i.e., $\bar{\mu} \circ T^{-1}=\bar{\mu}$, and is called the stationary mean of $\mu$. Every stationary measure is AMS [43]. Moreover, for an AMS measure $\mu$, the transported measure $\mu \circ\left(f^{\mathbb{Z}}\right)^{-1}$ is AMS under mild conditions, cf. [43, Example 6], 18]. The stationary mean $\overline{\mu \circ\left(f^{\mathbb{Z}}\right)^{-1}}$ will be called the stationary coding of measure $\mu$ w.r.t. function $f$.

Assuming that (64) is satisfied, this proposition has been proved in [18]:

Theorem 9 Let $\mu=P\left(\left(X_{i}\right)_{i \in \mathbb{Z}} \in \cdot\right)$ be the distribution of the process (5) where variables $K_{i}$ are IID and satisfy (7). Put $\mathbb{Y}=\{0,1,2\}$ and consider a function $f: \mathbb{X} \mapsto \mathbb{Y}^{+}$given as

$$
f(k, z)=b(k) z 2,
$$

where $1 b(k) \in\{0,1\}^{+}$is the binary representation of a natural number $k$. The process $\left(\bar{Y}_{i}\right)_{i \in \mathbb{Z}}$ distributed according to the stationary coding of measure $\mu$ w.r.t. function $f, \bar{P}\left(\left(\bar{Y}_{i}\right)_{i \in \mathbb{Z}} \in \cdot\right)=\overline{\mu \circ\left(f^{\mathbb{Z}}\right)^{-1}}$, satisfies conditions $(a)-(d)$ for $\zeta\left(\beta^{-1}\right)>4$. Variables $\bar{Z}_{k}$ may be constructed as $\bar{Z}_{k}=\bar{s}_{k}\left(\left(\bar{Y}_{i}\right)_{i \in \mathbb{Z}}\right)$ almost surely, where

$$
\bar{s}_{k}(w):= \begin{cases}0 & \text { if } 2 b(k) 02 \sqsubseteq w \text { and } 2 b(k) 12 \nsubseteq w, \\ 1 & \text { if } 2 b(k) 12 \sqsubseteq w \text { and } 2 b(k) 02 \nsubseteq w, \\ 2 & \text { else }\end{cases}
$$

for $w \in \mathbb{Y}^{\mathbb{Z}} \cup \mathbb{Y}^{*}$. 
Inequality $\zeta\left(\beta^{-1}\right)>4$ holds for $\beta>0.7728 \ldots$ and comes from satisfying condition (c). Processes $\left(\bar{Y}_{i}\right)_{i \in \mathbb{Z}}$ and $\left(X_{i}\right)_{i \in \mathbb{Z}}$ live on different probability spaces, say $(\Omega, \mathfrak{J}, \bar{P})$ and $(\Omega, \mathfrak{J}, P)$ respectively.

\section{A Excess-bounding lemma}

This proposition is a variation of a more specific statement in [15, Theorem 2]:

Lemma 1 Consider a function $G: \mathbb{N} \rightarrow \mathbb{R}$ such that $\lim _{k} G(k) / k=0$ and $G(n) \geq 0$ for all but finitely many $n$. For any $A \in \mathbb{N}$ and infinitely many $n$, we have $A G(n)-G(A n) \geq 0$.

Proof: We have the identity

$$
\sum_{k=0}^{m-1} \frac{A G\left(A^{k} n\right)-G\left(A^{k+1} n\right)}{A^{k+1}}=G(n)-n \cdot \frac{G\left(A^{m} n\right)}{A^{m} n} .
$$

For $m$ tending to infinity, this implies

$$
\sum_{k=0}^{\infty} \frac{A G\left(A^{k} n\right)-G\left(A^{k+1} n\right)}{A^{k+1}}=G(n) .
$$

Putting $n=A^{p}$, we obtain

$$
\sum_{k=p}^{\infty} \frac{A G\left(A^{k}\right)-G\left(A^{k+1}\right)}{A^{k+1}} \geq 0
$$

for all but finitely many $p$. On the other hand, if $A G(n)-G(A n) \geq 0$ did not hold for infinitely many $n$ then the sum in (69) would be strictly negative for all sufficiently large $p$.

Two specific applications of that lemma, namely implications (171) and (72), are used in this paper. Firstly, consider functions $G_{1}(n) \geq G_{2}(n) \geq 0$ and $F_{i}(n)=2 G_{i}(n)-G_{i}(2 n)$. If the functions $G_{i}$ have equal $\operatorname{limits} \lim _{n} G_{i}(n) / n=$ $g<\infty$ then

$$
\limsup _{n \rightarrow \infty}\left[F_{2}(n)-F_{1}(n)\right] \geq 0
$$

follows from Lemma 1. From inequality (70) and inequality $\lim \sup _{n}\left(a_{n}+b_{n}\right) \geq$ $\lim \sup _{n} a_{n}+\liminf _{n} b_{n}$, we obtain

$$
\liminf _{n \rightarrow \infty} \frac{F_{1}(n)}{n^{\beta}}>0 \Longrightarrow \limsup _{n \rightarrow \infty} \frac{F_{2}(n)}{n^{\beta}}>0 .
$$

Secondly, if $G_{1}(n)=g n+\tilde{G}_{1}(n)$ then

$$
\liminf _{n \rightarrow \infty} \frac{\tilde{G}_{1}(n)}{n^{\beta}}>0 \Longrightarrow \limsup _{n \rightarrow \infty} \frac{F_{2}(n)}{n^{\beta}}>0 .
$$

A brief justification of the latter statement is as follows. The left-hand side implies that $G(n)=G_{2}(n)-g n-B n^{\beta} \geq 0$ for all but finitely $n$ and a certain $B>0$. Then it suffices to apply Lemma 1 to obtain the right-hand side. 


\section{B A bound for the longest repeat}

Let us prove a bound for the maximal length of a repeat, defined in (28):

Lemma 2 For a finite-energy process $\left(X_{i}\right)_{i \in \mathbb{Z}}$ over a countable alphabet $\mathbb{X}$,

$$
\sup _{n \geq 2} \mathbf{E}\left(\frac{\mathbf{L}\left(X_{1: n}\right)}{\log n}\right)^{q}<\infty, \quad q>0 .
$$

Remark: The almost sure version of bound (73) was shown for finite-energy processes over a finite alphabet by Shields [25]. Such bound does not hold for stationary processes in general, cf. [44].

Proof: Assume (8) and consider a $j>i \geq 0$. Applying the idea from [45], let us notice that given $X_{1: j}=w$ condition $X_{j+1: j+k}=X_{i+1: i+k}$ is equivalent to $X_{j+1: j+k}=u$ for a certain string $u$ that is a function of $w$. Thus

$$
\begin{aligned}
& P\left(X_{j+1: j+k}=X_{i+1: i+k}\right) \\
& =\sum_{w \in \mathbb{X}^{j}} P\left(X_{j+1: j+k}=X_{i+1: i+k} \mid X_{1: j}=w\right) P\left(X_{1: j}=w\right) \\
& \leq \sum_{w \in \mathbb{X}^{j}} K c^{k} P\left(X_{1: j}=w\right)=K c^{k} .
\end{aligned}
$$

Hence

$$
\begin{aligned}
P\left(\mathbf{L}\left(X_{1: n}\right) \geq k\right) & =P\left(\exists_{0 \leq i<j \leq n-k} X_{j+1: j+k}=X_{i+1: i+k}\right) \\
& \leq \sum_{0 \leq i<j \leq n-k} P\left(X_{j+1: j+k}=X_{i+1: i+k}\right) \\
& \leq \frac{(n-k)(n-k-1)}{2} K c^{k} \leq \frac{n^{2} K c^{k}}{2} .
\end{aligned}
$$

This bound is nontrivial for $k>A:=(2 \log n+\log K-\log 2) / \log c^{-1}$. Consider a sufficiently large $n$ so that $A \geq 1$. Then inequality (73) follows from the series of inequalities

$$
\begin{aligned}
\mathbf{E}\left(\mathbf{L}\left(X_{1: n}\right)\right)^{q} & \leq A^{q}+\sum_{k>A} k^{q} P\left(\mathbf{L}\left(X_{1: n}\right) \geq k\right) \\
& \leq \sum_{k=0}^{\infty}(k+A)^{q} c^{k} \leq A^{q} \sum_{k=0}^{\infty}(k+1)^{q} c^{k},
\end{aligned}
$$

where $\sum_{k=0}^{\infty}(k+1)^{q} c^{k}<\infty$.

\section{Acknowledgment}

The author wishes to thank Peter Harremoës, Peter Grünwald, Alfonso Martinez, Jan Mielniczuk, Jacek Koronacki, Laurence Cantrill, Paul Vitanyi, En-hui Yang, and an anonymous referee for discussing the challenging composition of this paper. Special thanks are due to James Crutchfield, Arthur Ramer, and Peter Grünwald for inviting the author's to the Santa Fe Institute, the University of New South Wales, and the Centrum Wiskunde \& Informatica, where important constructions for this paper were completed. Finally, we thank cordially Prof. Emerit. Gabriel Altmann for many words of support during our investigations. 


\section{References}

[1] Ł. Dębowski, "On vocabulary size of grammar-based codes," in Proceedings of 2007 IEEE International Symposium on Information Theory. Nice, France, July 25-29, 2007, pp. 91-95.

[2] G. K. Zipf, The Psycho-Biology of Language: An Introduction to Dynamic Philology, 2nd ed. The MIT Press, 1965.

[3] J. C. Kieffer and E. Yang, "Grammar-based codes: A new class of universal lossless source codes," IEEE Trans. Inform. Theor., vol. 46, pp. 737-754, 2000.

[4] M. Charikar, E. Lehman, A. Lehman, D. Liu, R. Panigrahy, M. Prabhakaran, A. Sahai, and A. Shelat, "The smallest grammar problem," IEEE Trans. Inform. Theor., vol. 51, pp. 2554-2576, 2005.

[5] B. Mandelbrot, "Structure formelle des textes et communication," Word, vol. 10, pp. 1-27, 1954.

[6] G. A. Miller, "Some effects of intermittent silence," Amer. J. Psych., vol. 70, pp. 311-314, 1957.

[7] H. A. Simon, "On a class of skew distribution functions," Biometrika, vol. 42, pp. 425-440, 1955.

[8] P. Harremoës and F. Topsøe, "Maximum entropy fundamentals," Entropy, vol. 3, pp. 191-226, 2001.

[9] W. Kuraszkiewicz and J. Łukaszewicz, "The number of different words as a function of text length," Pamiętnik Literacki, vol. 42(1), pp. 168-182, 1951, in Polish.

[10] P. Guiraud, Les caractères statistiques du vocabulaire. Paris: Presses Universitaires de France, 1954.

[11] G. Herdan, Quantitative Linguistics. Butterworths, 1964.

[12] H. S. Heaps, Information Retrieval-Computational and Theoretical Aspects. Academic Press, 1978.

[13] E. Khmaladze, "The statistical analysis of large number of rare events," 1988, Technical Report MS-R8804. Centrum voor Wiskunde en Informatica, Amsterdam.

[14] A. Kornai, "How many words are there?" Glottometrics, vol. 4, pp. 61-86, 2002.

[15] Ł. Dębowski, "On Hilberg's law and its links with Guiraud's law," J. Quantit. Linguist., vol. 13, pp. 81-109, 2006.

[16] _ " "Menzerath's law for the smallest grammars," in Exact Methods in the Study of Language and Text, P. Grzybek and R. Köhler, Eds. Mouton de Gruyter, 2007, pp. 77-85. 
[17] — "A general definition of conditional information and its application to ergodic decomposition," Statist. Probab. Lett., vol. 79, pp. 1260-1268, 2009 .

[18] — , "Variable-length coding of two-sided asymptotically mean stationary measures," J. Theor. Probab., vol. 23, pp. 237-256, 2010.

[19] J. G. Wolff, "Language acquisition and the discovery of phrase structure," Lang. Speech, vol. 23, pp. 255-269, 1980.

[20] C. G. de Marcken, "Unsupervised language acquisition," Ph.D. dissertation, Massachussetts Institute of Technology, 1996.

[21] C. Kit and Y. Wilks, "Unsupervised learning of word boundary with description length gain," in Proceedings of the Computational Natural Language Learning ACL Workshop, Bergen, M. Osborne and E. T. K. Sang, Eds., 1999, pp. 1-6.

[22] C. G. Nevill-Manning, "Inferring sequential structure," Ph.D. dissertation, University of Waikato, 1996.

[23] G. J. Chaitin, "A theory of program size formally identical to information theory," J. ACM, vol. 22, pp. 329-340, 1975.

[24] M. Gardner, "The random number $\Omega$ bids fair to hold the mysteries of the universe," Sci. Am., vol. 241, pp. 20-34, 1979.

[25] P. C. Shields, "String matching bounds via coding," Ann. Probab., vol. 25, pp. 329-336, 1997.

[26] J. P. Crutchfield and D. P. Feldman, "Regularities unseen, randomness observed: The entropy convergence hierarchy," Chaos, vol. 15, pp. 25-54, 2003.

[27] W. Hilberg, "Der bekannte Grenzwert der redundanzfreien Information in Texten - eine Fehlinterpretation der Shannonschen Experimente?" Frequenz, vol. 44, pp. 243-248, 1990.

[28] C. Shannon, "Prediction and entropy of printed English," Bell Syst. Tech. J., vol. 30, pp. 50-64, 1950.

[29] W. Ebeling and G. Nicolis, "Entropy of symbolic sequences: the role of correlations," Europhys. Lett., vol. 14, pp. 191-196, 1991.

[30] W. Ebeling and T. Pöschel, "Entropy and long-range correlations in literary English," Europhys. Lett., vol. 26, pp. 241-246, 1994.

[31] W. Bialek, I. Nemenman, and N. Tishby, "Complexity through nonextensivity," Physica A, vol. 302, pp. 89-99, 2001.

[32] T. M. Cover and J. A. Thomas, Elements of Information Theory. Wiley, 1991.

[33] D. Neuhoff and P. C. Shields, "Simplistic universal coding," IEEE Trans. Inform. Theor., vol. IT-44, pp. 778-781, 1998. 
[34] P. Elias, "Universal codeword sets and representations of the integers," IEEE Trans. Inform. Theor., vol. 21, pp. 194-203, 1975.

[35] T. Weissman, "Not all universal source codes are pointwise universal," 2004, http://www.stanford.edu/ ${ }^{\sim}$ tsachy/interest.htm.

[36] O. Kallenberg, Foundations of Modern Probability. Springer, 1997.

[37] R. M. Gray and L. D. Davisson, "The ergodic decomposition of stationary discrete random processses," IEEE Trans. Inform. Theor., vol. 20, pp. 625636, 1974.

[38] D. S. Ornstein and B. Weiss, "How sampling reveals a process," Ann. Probab., vol. 18, pp. 905-930, 1990.

[39] I. M. Gelfand, A. N. Kolmogorov, and A. M. Yaglom, "Towards the general definition of the amount of information," Dokl. Akad. Nauk SSSR, vol. 111, pp. 745-748, 1956, in Russian.

[40] P. Billingsley, Probability and Measure. Wiley, 1979.

[41] R. L. Dobrushin, "A general formulation of the fundamental Shannon theorems in information theory," Usp. Matem. Nauk, vol. 14(6), pp. 3-104, 1959 , in Russian.

[42] M. S. Pinsker, Information and Information Stability of Random Variables and Processes. Holden-Day, 1964.

[43] R. M. Gray and J. C. Kieffer, "Asymptotically mean stationary measures," Ann. Probab., vol. 8, pp. 962-973, 1980.

[44] P. C. Shields, "String matching: The ergodic case," Ann. Probab., vol. 20, pp. 1199-1203, 1992.

[45] I. Kontoyiannis and Y. Suhov, "Prefixes and the entropy rate for longrange sources," in Probability Statistics and Optimization: A Tribute to Peter Whittle, F. Kelly, Ed. Wiley, 1994, pp. 89-98. 